\title{
Some ELF Phenomena ${ }^{1}$
}

\author{
E. T. Pierce
}

(February 23, 1960)

\begin{abstract}
Properties of the electric and magnetic fields in natural extremely low frequency (ELF) phenomena are briefly discussed. The ELF fluctuations in the electric field are then treated from two aspects; these are the electromagnetic changes associated with atmospherics and the electrostatic variations in atmospheric electricity. A final section attempts to integrate the general subject of ELF effects of natural origin.
\end{abstract}

\section{Introduction}

Any antenna arrangement for receiving signals of natural origin may be envisaged as responding either to the electric $(E)$ field or to the magnetic $(H)$ field. An example of the first instance would be a vertical whip, and of the second an electrostatically shielded loop antenna. The $E$ field has three components $E=E_{S}+E_{I}+E_{R}$ and the $H$ field two terms $H=H_{I}+$ $H_{R}$.

The individual components with suffix $R$ are normally described as "radiation" or "electromagnetic"; those with the I suffix are termed "induction," "magnetic," or "current"; while the "electrostatic" component has the suffix $S . H_{I}$ and $E_{I}$ are, of course, interrelated, as are $E_{R}$ and $H_{R}$; also, in free space the $S, I$, and $R$ terms are proportional respectively to $1 / d^{3}, 1 / d^{2}$, and $1 / d$, where $d$ is the distance from the source to the receiver. Furthermore, if the source is considered to radiate at a discrete frequency, as in the case of an oscillating dipole then

$$
\left.\begin{array}{c}
E_{S}: E_{I}: E_{R}=1: \frac{\omega d}{c}: \frac{\omega^{2} d^{2}}{c^{2}} \\
H_{I}: H_{R}=1: \frac{\omega d}{c}
\end{array}\right\}
$$

where $\omega$ is the angular frequency concerned and $c$ is the velocity of light. It is evident from these relations that for extremely low frequencies, less than say $1 \mathrm{kc}$, the electrostatic component is dominant in the electric field even at considerable distances from the source. However, it should be remembered that in actual practice propagation is not unrestricted as in free space but confined, typically between the earth and the lower ionosphere, and when boundary conditions have to be applied the relations among the different field components are inevitably modified. ${ }^{2}$

1 Contribution from AVCO Researeh and Advanced Development Div. Wilmington, Mass : paper presented at Conference on the Propagation of ELF Radio Waves, Boulder, Colo., Jan. 25, 1960.

2 See paper by J. R. Wait in this issue for such an analysis.
Many aspects of ELF phenomena have been studied in the past. Two of these are considered in the succeeding sections, and some final discussion then attempts to integrate the whole field of ELF effects.

\section{The "Slow Tail"}

The radiation fields due to distant lightning or "atmospherics" as they are more commonly termed, often contain both ELF ( $<1 \mathrm{kc}$ ) and VLF (ca $10 \mathrm{kc}$ ) components. This effect is basically due to the pronounced propagational attenuation at around 1 to 3 $\mathrm{kc}$; this has been established both experimentally [1] and theoretically [2]. A typical waveform of distant origin is depicted in figure 1 . It is of an atmospheric due to a discharge to earth. The 10msec sweep is initiated by a precursor; then follows the disturbance, some $300 \mathrm{mv} / \mathrm{m}$ peak-to-peak, known variously as the "VLF section," the "oscillatory head," or the "main stroke"; and this is succeeded by the "ELF portion" or "slow tail." Watson-Watt, Herd, and Lutkin [3] were the first to make a detailed study of the ELF section; they noticed the important feature that the farther away the origin of the atmospheric the greater the time separation $t$ between the oscillatory head and the slow tail. Hales [4] applied the waveguide theory of propagation between the earth and a sharply bounded lower ionosphere to the ELF results of Watson-Watt and his associates. Hales was primarily concerned with the quasi zero-order mode. Hepburn and Pierce [5] established experimentally the following empirical laws relating $t, d$, and $\tau / 4$, the time occupied by the first quarter cycle of the slow tail:

$$
\begin{array}{r}
\text { day }\left\{\begin{array}{l}
\frac{\tau}{4}=500+0.23 d \\
t=0.33 d-350
\end{array}\right. \\
\operatorname{night}\left\{\begin{array}{l}
\frac{\tau}{4}=500+0.08 d \\
t=0.13 d-140 .
\end{array}\right.
\end{array}
$$




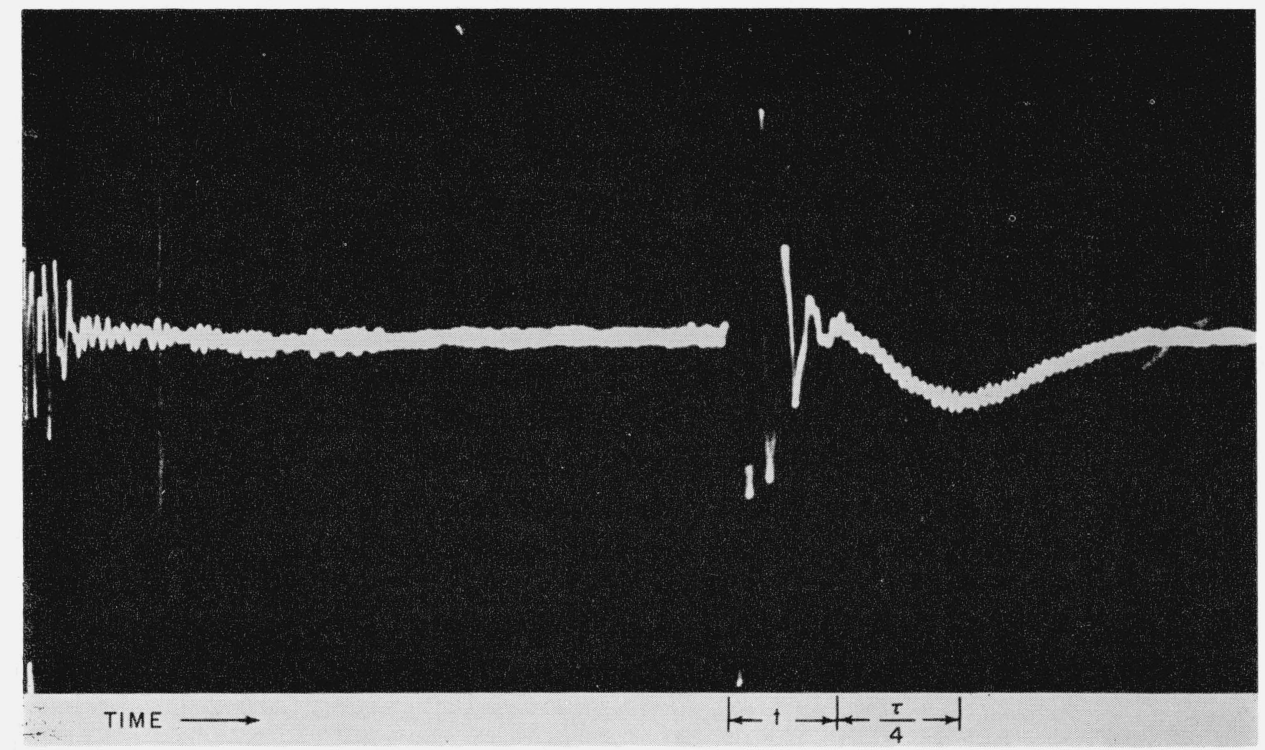

Figure 1. A typical waveform.

The units are kilometers for $d$ and microseconds for $t$ and $\tau$.

Using the theory developed by Hales, Hepburn, and Pierce were able to deduce from their experimental results a range of pairs of solutions for $h$ the height of the ionospheric stratum and $\sigma$ its conductivity. Neither can be determined separately but the most likely solution can be assessed since there is other information available on the variation of electron density $N$ and collisional frequency $\nu$ with height $h$, and therefore of $\sigma$, since this is mainly dependent on the ratio $N / \nu$. The final result of Hepburn and Pierce was that the experimental observations by day indicated an ionospheric layer of height $65 \mathrm{~km}$ and conductivity $5 \times 10^{3} \mathrm{esu}$; at night the corresponding figures were $90 \mathrm{~km}$ and $10^{4}$ esu. Hepburn [6] in a revised analysis later gave $70 \mathrm{~km}$ and $2 \times 10^{3}$ esu for day, and $90 \mathrm{~km}$ and $4 \times 10^{3}$ esu at night.

Liebermann [7] has also worked on slow tails. He derived his own theory which produced the relation

$$
d=4 h(\pi \sigma \tau)^{1 / 2} .
$$

Here the units are kilometers for $d, h$ and seconds for $\tau$. Liebermann found that his experimental results fitted his theoretical relationship with values of $h$ and $\sigma$ of 60 and $10^{4}$ esu by day; at night the figures were 90 and $5 \times 10^{4}$. There is obviously some discordance between the work of Liebermann and that of Hepburn and Pierce; it has not yet been resolved.

The question of the generation of slow tails is of some interest. It seems certain that many tails arise from a continuing current following the return stroke in a flash to earth. Pierce [8] in his observations of the electrostatic field-changes associated with close discharges has pointed out that some flashes seem to have a pronounced slow change of field after the return stroke; in other discharges this slow change appears to be absent. Thus there should be a tendency for some flashes to give large slow tails while others produce little or no ELF component. Hepburn, however, believes that the distribution is relatively continuous. In an effort to resolve this point a histogram (fig. 2) has been plotted of the ELF/VLF ratios for several hundred atmospherics recently recorded at Avco, Wilmington, Mass. The great majority of these, being winter atmospherics, tended to originate at comparable distances. The histogram indicates that the distribution is much more extended than would be expected from the smaller values of the ELF/VLF ratios, or, in other words, the contention that certain families of lightning flashes are extremely potent generators at ELF's seems to be supported. In this connection it is perhaps noteworthy that cloud discharges may produce strong ELF signals; if so, they would be expected to give an ELF to VLF ratio appreciably different from that in flashes to earth.

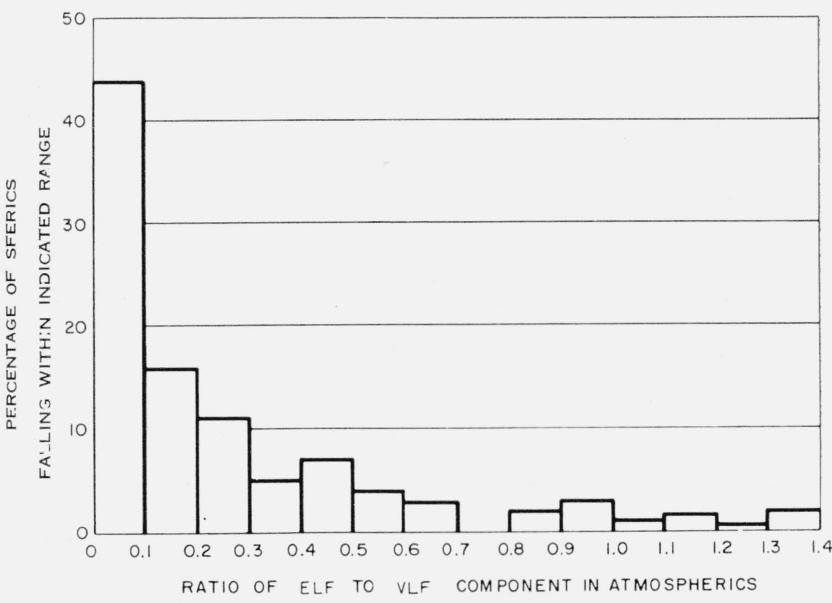

FIGURE 2. Histogram illustrating the statistical behavior of the $E L F / V L F$ ratio for atmospherics. 


\section{ELF Signals in Atmospheric Electricity}

In atmospheric electricity as compared with atmospherics, the ELF signals are predominantly electrostatic rather than electromagnetic. The work of Holzer and his school [9] represents a natural bridge in relating the two separate fields. It is well known that the electrostatic field changes accompanying a lightning flash often have durations approaching a second, and that violent fluctuations of the atmospheric potential gradient due to rain, point-discharge, space charge movements, and so on, occur in disturbed weather. These effects produce a spectrum of ELF phenomena extending both well above and well below 1 cps. Appreciable fluctuations at ELF's can, however, also occur in fair weather. Examples have been noted by Pierce [8] and one is reproduced in figure 3 . The record is from a capillary electrometer; the total duration is 35 sec and the peak-to-peak amplitudes of the electrostatic fluctuations in potential gradient are of the order of $1 \mathrm{v} / \mathrm{m}$.

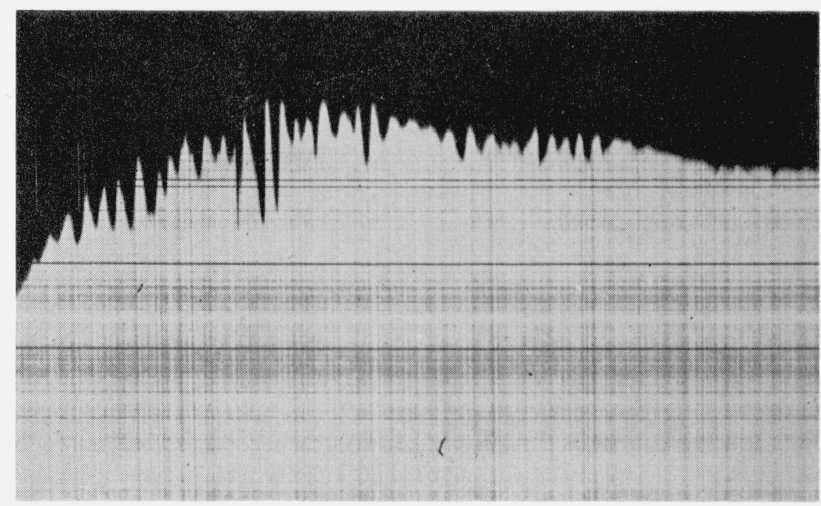

Figure 3. ELF fluctuations in the fair weather electrostatic field.

Large $[10,11]$ has made a detailed investigation of the behavior along the spectrum of variations in the electric field. He used experimental methods of recording simultaneously on two spaced aerials in order to separate the effects of local and of distant origin; the frequency range from $10 \mathrm{kc}$ downwards was covered with broadband and with sharply tuned receivers. Large believes that because the natural relaxation or readjustment time of the lower atmosphere is of the order of $10^{3} \mathrm{sec}$, such effects as changes in resistivity due to altering pollution will produce variations with frequencies less than $10^{-3} \mathrm{cps}$. From $10^{-3}$ to 1 cps Large finds that fair weather variations are due to the movement of space charges. These often travel with the wind at a height of 10 or $20 \mathrm{~m}$. There is also a turbulent component which is well correlated with the diurnal variation of turbulence. Above 1 cps the situation is somewhat confused but the general picture seems to be the following. From $1 \mathrm{kc}$ to 100 cps disturbances tend to be electromagnetic being the discrete slow tails well correlated with VLF atmospherics at $10 \mathrm{kc}$. Below $100 \mathrm{cps}$ the correlation with $10 \mathrm{kc}$ becomes less and less marked and the fluctuations not discrete but more and more continuous. This is for two reasons. Propagation at $100 \mathrm{cps}$ is already better even than at $10 \mathrm{kc}$ and is becoming increasingly good from 100 cps downwards; thus ever more distant sources of atmospherics are being detected, and the individual disturbances are therefore becoming increasingly frequent, that is, approaching a continuous noise. Also, with a decrease in frequency, the electrostatic fluctuations due to the movement of space charges are becoming more and more marked, and Large believes that they are the dominant factor for frequencies less than some $20 \mathrm{cps}$.

\section{Discussion}

Some kind of summary can now be made of the behavior of both the electric and magnetic fields for frequencies below $1 \mathrm{kc}$. As Large has shown the $E$ field changes over from being dominantly electromagnetic and originating in distant lightning flashes, to being mainly electrostatic with a source in local movements of space charge. The changeover is at a frequency of about 20 cps. Below 20 cps the phenomena are almost entirely electrostatic, but at "extremely low frequencies indeed" the origin is no longer necessarily local. For instance with frequencies of the order of $10^{-4}$ to $10^{-5}$ cps the diurnal variations of local atmospheric pollution and of worldwide thunderstorm activity are both effective as regards changes in the atmospheric potential gradient. At $10^{-7}$ cps seasonal influences are entering and there are further possibilities of annual and longer duration variations.

The behavior of the $H$ field is in some respects similar to that of the $E$ field. Aarons [12] has pointed out that there is probably a changeover in dominance from the electromagnetic to the magnetic component at about $20 \mathrm{cps}$. Below this frequency the main features are caused by magnetic effects due to ionospheric and earth currents. Thus at the higher frequencies the $E$ and $H$ fields are both mostly electromagnetic and have a common origin in distant lightning flashes. Below about $20 \mathrm{cps}$ the $E$ field is predominantly electrostatic and the $H$ field mostly magnetic; their sources, moreover, are entirely distinct. Therefore $E$ and $H$ measurements should correlate at the higher frequencies and be increasingly unrelated as frequency decreases. This is entirely the kind of behavior found when the work of Willis [13], who used loop antenna responding to the $H$ field, is compared with the $E$ measurements of Large.

\section{References}

[1] F. W. Chapman and R. C. V. Macario, Propagation of audio-frequency radio waves to great distances, Nature (London) 17\%, 930 (1956).

[2] J. R. Wait, The attenuation vs frequency characteristies of VLF radio waves, Proc. IRE 45, 768 (1957).

[3] R. A. Watson-Watt, J. F. Herd, and F. E. Lutkin, On the nature of atmospherics, Proc. Roy. Soc. [A] 162, 267 (1937). 
[4] A. L. Hales, A possible mode of propagation of the slow or tail components in atmospherics, Proc. Roy. Soc. [A] 193, 60 (1948).

[5] F. Hepburn and E. T. Pierce, Atmospherics with very low-frequency components, Nature (London) 171, 83 ? (1953).

[6] F. Hepburn, Atmospheric waveforms with very low freauency components below $1 \mathrm{kc} / \mathrm{s}$ known as slow tails, J. Atmos. Terr. Phys. 10, 266 (1957).

[7] L. Liebermann, Extremely low-frequency electromagnetic waves. II. Propagation-properties, J. Appl. Phys. 27, 1477 (1956).

[8] E. T. Pierce, Electrostatic field-changes due to lightning discharges, Quart. J. Roy. Met. Soc. 81, 211 (1955).

[C] R. E. Holzer and O. E. Deal, Low audio-frequency electromagnetic signals of natural origin, Nature (London) 18\%, 536 (1956).
[10] M. I. Large, Some investigations in atmospheric electricity, Dissertation (Cambridge Univ., Cambridge, England, 1957).

[11] M. I. Large and T. W. Wormell, Fluctuations in the vertical electric field, Recent Adv. Atmos. Elec., p. 603 (Pergamon Press, Ltd., New York, N.Y., 1958).

[12] J. Aarons, Low frequency electromagnetic radiation 10-900 cycles per second, J. Geophys. Research 61, 647 (1956).

[13] H. F. Willis, Audio-frequency magnetic fluctuations, Nature (London) 161, 887 (1948). 